\title{
The interaction of artemisinin with malarial hemozoin
}

\author{
Yu-Long Hong, Ying-Zi Yang, Steven R. Meshnick* \\ Department of Epidemiology, University of Michigan School of Public Health, 109 Observatory Street, Ann Arbor, MI 48109, USA
}

(Received 7 September 1993; accepted 28 October 1993)

\begin{abstract}
$\left[{ }^{14} \mathrm{C}\right]$ Artemisinin was taken up by Plasmodium falciparum in culture and concentrated in hemozoin. In vitro, hemin and artemisinin were found to undergo a chemical reaction forming two major products which were isolated by highperformance liquid chromatography (HPLC). The $m / z$ values of the two products were 856 and 871 . Thin-layer chromatography (TLC) and HPLC of hemozoin isolated from $\left[{ }^{14} \mathrm{C}\right]$ artemisinin-treated parasites showed that the majority of the hemozoin-associated radioactivity comigrated with the synthetic adducts. When $\left[{ }^{14} \mathrm{C}\right] \operatorname{artemisinin}$ was incubated with isolated hemozoin, $\left[{ }^{14} \mathrm{C}\right]$ artemisinin disappeared from the solution in a time-dependent manner. Some of the radioactivity present in the treated hemozoin also comigrated with the adducts on TLC. Thus, artemisinin appears to react covalently with heme in malaria hemozoin both in vitro and in situ.
\end{abstract}

Key words: Antimalarial; Qinghaosu; Malaria; Endoperoxide; Plasmodium; Haem; Alkylation

\section{Introduction}

Artemisinin, which is derived from an ancient Chinese herbal remedy for fever, is a sesquiterpene endoperoxide $[1,2]$. Several artemisinin derivatives, especially artemether and artesunate have been widely used in Asia, particularly China, where over one million patients have been treated [3]. In addition, a large number of synthetic endoperoxides have been synthesized and found to

"Corresponding author. Tel.: (313)747-2406; Fax: (313)7643192. E-mail: meshnick@umich.edu.

Abbreviations: ESI, electrospray ionization; FAB, fast atom bombardment; HPLC, high-performance liquid chromatography; TLC, thin-layer chromatography. have antimalarial activity. One of these synthetic endoperoxides, Ro. 42-1611, is currently undergoing Phase II clinical trials ( $R$. Ridley, Hoffman-LaRoche, personal communication). Thus, the artemisinin derivatives represent a promising new class of antimalarial agents.

Malaria parasites live inside erythrocytes and consume hemoglobin. When heme molecules are released, they polymerize and accumulate in intraparasitic granules known as malaria pigment or hemozoin $[4,5]$. We have previously proposed that this accumulation of heme sensitizes malaria parasite to the toxic effects of artemisinin and its derivatives, because it catalyzes the decomposition of the drug into free radicals which act as alkylating agents [6-9]. In this paper we show that artemisinin concentrates in hemozoin where it is cova- 
lently bound to heme.

\section{Materials and methods}

2.1. Chemicals. Artemisinin was purchased from Aldrich Chemical Company (Milwaukee, WI). $\left[{ }^{14} \mathrm{C}\right]$ Artemisinin $\left(45.5 \mathrm{mCi} \mathrm{mmol}^{-1}\right)$ was a gift from the Division of Experimental Therapeutics, Walter Reed Army Institute of Research, Washington DC. All other chemicals were purchased from Sigma Chemical Co. (St. Louis, MO).

\subsection{Isolation of malarial hemozoin. P. falciparum} FCR3 strain was cultivated by the method of Trager and Jensen [10]. Hemozoin was isolated from these cultures by a modification of the method of Goldie et al. [11]. Parasite cultures were pooled and the cells were pelleted by centrifugation at $540 \times g$. The pellet was washed once with RPMI and then lysed by the addition of an equal volume of hypotonic buffer $(8 \mathrm{mM}$ potassium phosphate/ $9 \mathrm{mM}$ disodium EDTA, $\mathrm{pH}$ 7.2) followed by sonication with a microprobe at maximum intensity for $15 \mathrm{~s}$ (Sonifier Cell Disruptor, Heat SystemsUltrasonics, Long island, NY). Crude hemozoin was then pelleted by centrifugation at $35000 \times g$ at $4{ }^{\circ} \mathrm{C}$ for $10 \mathrm{~min}$. The pellet was resuspended in $10 \mathrm{ml}$ hypotonic buffer, sonicated and centrifuged at least thrice, until the supernatant became colorless. The pellet was then resuspended in $1 \mathrm{ml}$ hypotonic buffer and carefully layered on top of 10 $\mathrm{ml}$ of $30 \%$ sucrose in hypotonic buffer and centrifuged at $35000 \times \mathrm{g}$ for $1 \mathrm{~h}$ at $4{ }^{\circ} \mathrm{C}$. The hemozoin, at the bottom of the gradient, was removed, washed 3 times with water, resuspended in $1 \mathrm{ml}$ $1 \%$ SDS and centrifuged at $13000 \times g$. The pellet and pooled supernatants were stored at $-80^{\circ} \mathrm{C}$.

\subsection{Synthesis and partial isolation of hemin $\backslash$ artem-} isinin adducts. A $10 \mathrm{mM}$ hemin solution was prepared immediately prior to each experiment by first dissolving hemin in $1 \mathrm{~N} \mathrm{NaOH}$, diluting with Tris- $\mathrm{HCl}$ buffer $(50 \mathrm{mM} \mathrm{pH} 8.5)$ and adjusting the $\mathrm{pH}$ to 8.5 with $1 \mathrm{~N} \mathrm{HCl}$. Artemisinin was dissolved in methanol as a $10 \mathrm{mM}$ solution and stored at $-20^{\circ} \mathrm{C}$. Equal volumes of hemin $(10$
$\mathrm{mM})$ and artemisinin $(10 \mathrm{mM})$ were mixed and incubated in the dark at $37^{\circ} \mathrm{C}$ for $20-24 \mathrm{~h}$.

The products of the reaction were separated by using high-performance liquid chromatography (HPLC; Rainin Instrument Co. Inc., Woburn, $\mathrm{MA}$ ) with a $300 \times 22.5 \mathrm{~mm}$ Bondclone $\mathrm{C} 18$ semipreparative column (Phenomenex, Torrance, CA) and a flow rate of $15 \mathrm{ml} \mathrm{min}^{-1}$ by a modification of the methods of Choe and Ortiz de Montellano [12]. The column was eluted with a mixture of methanol/ water/ acetic acid (32:18:5) and monitored at $400 \mathrm{~nm}$. Before each injection, $600 \mu \mathrm{l}$ of the reaction mixture was mixed with $400 \mu \mathrm{l}$ of methanol/ acetic acid (3:1), clarified by centrifugation and injected. The products collected from the column were concentrated by low pressure distillation using a Buchi Rotary Evaporator (Brinkmann Instruments, Inc., Westbury, NY) until almost dry. The products were redissolved in methanol and rechromatographed by using a mixture of methanol/ water/acetic acid (7:3:1) as the elution buffer and concentrated as above. Finally the purified products were dissolved in methanol and stored at $-20^{\circ} \mathrm{C}$.

2.4. Mass spectroscopy. The molecular weights of the isolated reaction products were determined by two mass spectroscopic methods. Electrospray ionization (ESI) mass spectra were obtained on a model 201 single quadrupole mass spectrometer (Vestec Corp. Houston, TX), with a $2000 \mathrm{~m} / \mathrm{z}$ range and a Vestec electrospray source. Fast atom bombardment (FAB) ionization mass spectra was obtained on a model 70-250-S mass spectrometer (VG Analytical, Manchester, England), using a fast atom bombardment atom gun with a current of $1 \mathrm{~mA}$ and a voltage of $8 \mathrm{kV}$. Xenon was the gas ion source.

2.5. The reaction of isolated hemozoin and $\left[{ }^{14} \mathrm{C}\right]$ artemisinin. Hemozoin, which was isolated as above from 10 plates of untreated parasites, was incubated with $0.042 \mu \mathrm{Ci}\left[{ }^{14} \mathrm{C}\right]$ artemisinin in $1 \mathrm{ml}$ of $10 \%$ methanol and $90 \%$ PBS at $37{ }^{\circ} \mathrm{C}$ in the dark. As a control, $0.042 \mu \mathrm{Ci}$ artemisinin was dissolved in the same solution in the absence of hemozoin. $7 \mu$ of supernatant were removed at 0,1 , 2 and $19 \mathrm{~h}$, respectively. 
2.6. Fate of radioactivity in drug-treated parasites. Hemozoin, which was isolated from $\left[{ }^{14} \mathrm{C}\right]$ artemisinin-treated parasites or was incubated with $\left[{ }^{14} \mathrm{C}\right]$ artemisinin as above, was dissolved in 200-300 $\mu 10.1 \quad \mathrm{~N} \mathrm{KOH}$ and then precipitated by the addition of one drop of $1 \mathrm{~N}$ $\mathrm{HCl}$. After microfuging for $1 \mathrm{~min}$, the supernatant was discarded and the pellet was redissolved in a mixture of methanol/ water/ acetic acid (32:18:5). $20 \mu \mathrm{l}$ were spotted onto a $\mathrm{C} 18$ reversephase fluorescence thin-layer chromatography (TLC) plate (Whatman International Ltd., Maidstone, England) and developed at room temparature with water/acetic acid/methanol (2:1:7). Artemisinin, hemin and the artemisinin-heme adducts isolated from HPLC were used as standards. After each run, the reverse phase plate layer was divided into $0.5-\mathrm{cm}$ fractions and scraped into scintillation vials. $100 \mu 1$ of methanol was added to dissolve the drug followed by $10 \mathrm{ml}$ of scintillation fluid. The radioactivity was then counted in a scintillation counter.

Alternatively, hemozoin was dissolved in $0.2 \mathrm{~N}$ $\mathrm{KOH}$, mixed with an equal volume of methanol/ acetic acid (9:1) and analyzed by HPLC on a 300 $\times 3.9 \mathrm{~mm} \mu$ Bondapak $\mathrm{C} 18$ analytical column (Waters, Milford MA), eluting at a flow rate of 1 $\mathrm{ml} \mathrm{min}^{-1}$ with methanol/water/acetic acid $(57: 34: 9)$ for $30 \mathrm{~min}$ followed by methanol/acetic acid $(9: 1)$ for $10 \mathrm{~min}$.

2.7. Biochemical assays. Protein in each fraction was determined by bicinchoninic acid binding [13]. Heme was determined by the modified methods of Fuhrhop and Smith [14]. Radioactivity was measured on an LKB 1219 Rackbeta or a Bechman LS 7000 Scintillation counter using Scintivers BD scintillation fluid (Fisher Scientific, Fair Lawn, NJ).

\section{Results}

When malaria parasites were incubated in the presence of $\left[{ }^{14} \mathrm{C}\right]$ artemisinin, more than $40 \%$ of the added drug was taken up by the cultures (data not shown). Of the cell-associated radioactivity, only $25 \%$ was found in the pooled washes
Table 1

Fate of radioactivity in $\left[{ }^{14} \mathrm{C}\right]$ artemisinin treated parasites ${ }^{\mathrm{a}}$

\begin{tabular}{lcrrr}
\hline Fraction & $\begin{array}{l}\text { Counts } \\
\text { min }\end{array}$ & $\%$ & \multicolumn{1}{l}{$\begin{array}{l}\text { Protein } \\
(\mathrm{mg})\end{array}$} & $\%$ \\
\hline $\begin{array}{l}\text { Pooled supernatants } \\
\text { Hemazoin }\end{array}$ & 84635 & 25 & 2081.3 & 99.58 \\
$\quad$ SDS-extract & 90665 & 27 & 8.3 & 0.40 \\
$\quad$ SDS-pellet & 165000 & 48 & 0.5 & 0.02 \\
Total & 340300 & & 2090.1 & \\
\hline
\end{tabular}

${ }^{\mathrm{a}}$ Cultured for $8 \mathrm{~h}$ in the presence of $\left[{ }^{14} \mathrm{C}\right.$ artemisinin $(2.7 \mathrm{nCi}$ $\left.\mathrm{ml}^{-1}\right)$.

and gradient, which contained more than $99 \%$ of the total protein (Table 1). The remainder, $75 \%$ of the radioactivity, was associated with the isolated parasite hemozoin. About $1 / 3$ of the hemozoinassociated radioactivity could be extracted with $1 \%$ SDS, which solubilized $>95 \%$ of the pelleted protein (Table 1). The SDS-washed hemozoin pel-

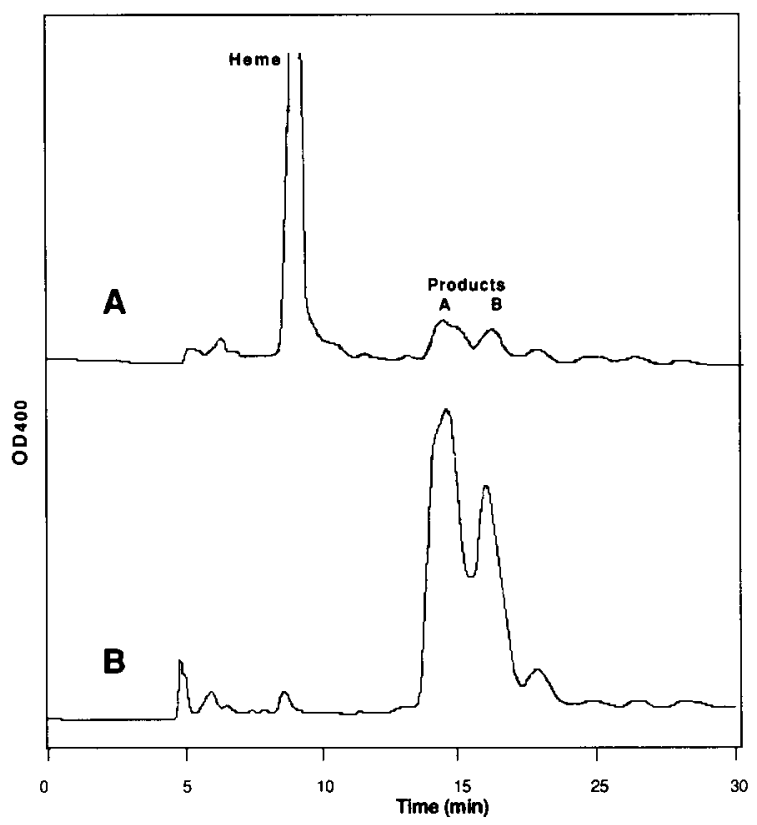

Fig. 1. HPLC separation of hemin and artemisinin reaction products after incubating $20 \mathrm{~h}$ at $37^{\circ} \mathrm{C}$ in the dark. HPLC was performed on a semipreparative column as described in the Materials and Methods, eluting with methanol/ water/ acetic acid (7:3:1). (A) Reaction mixture; (B) Rechromatography of pooled products from $A$. 
let contained the equivalent of $2.3 \mathrm{mmol}$ artemisinin (mol heme) $)^{-1}$.

In order to understand the interaction of hemin and artemisinin, the two compounds were mixed and allowed to react in solution. Two reaction product peaks were found by HPLC which eluted after the heme peak (Fig. 1). These peaks were not seen when either reactant was incubated in the same buffer but in the absence of the second reactant (data not shown). The molecular weights of the adducts were then determined by ESI and FAB mass spectroscopy. Both methods gave identical $m / z$ values for heme (616), adduct $\mathrm{A}$ (856), and adduct B (871) (Fig. 2). Hemin, artemisinin and the adducts could also be separated by reverse-phase TLC (Fig. 3). [The Rf of artemisinin
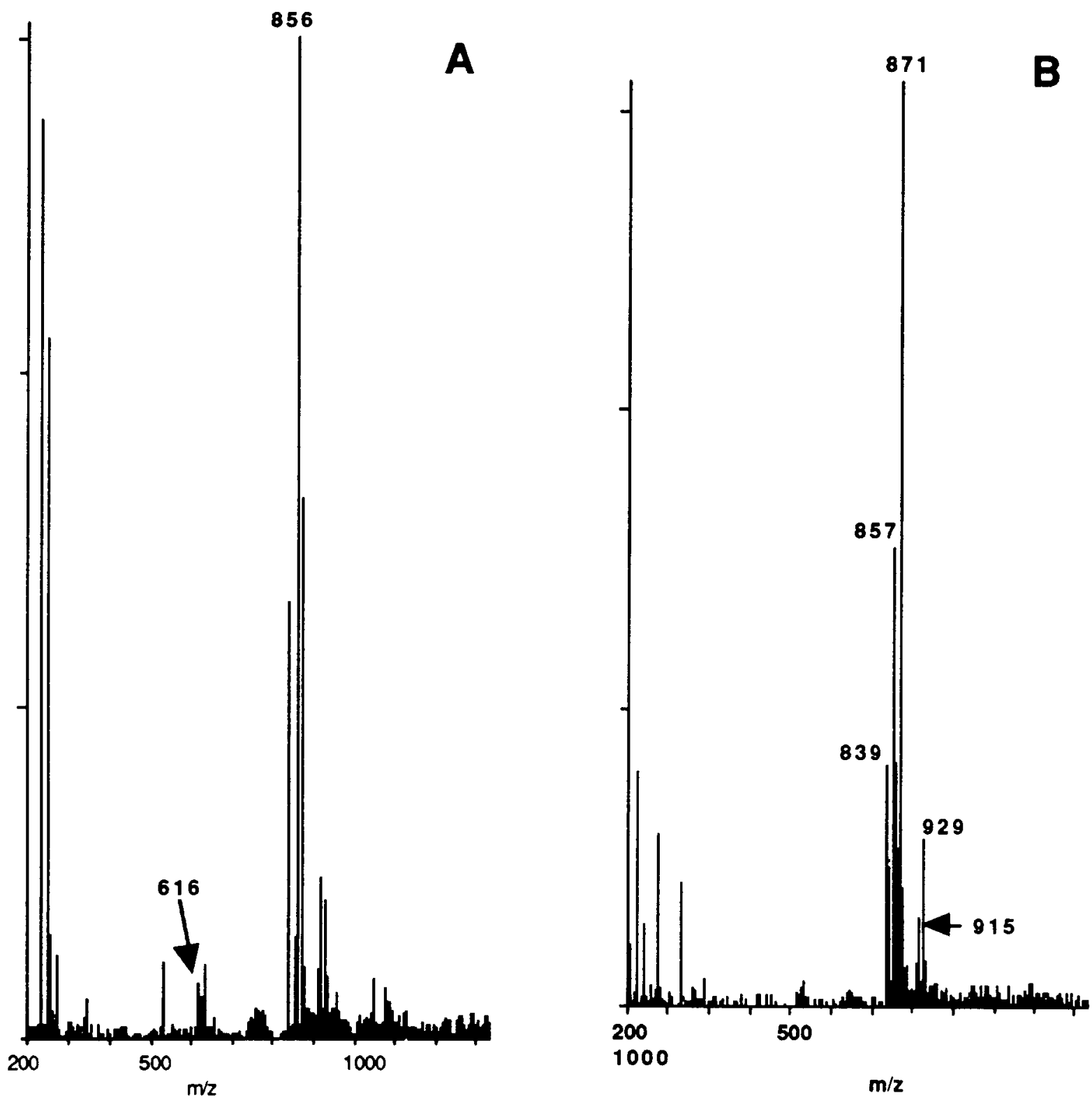

Fig. 2. Electrospray mass spectra of HPLC-purified hemin-artemisinin reaction products A and B. 


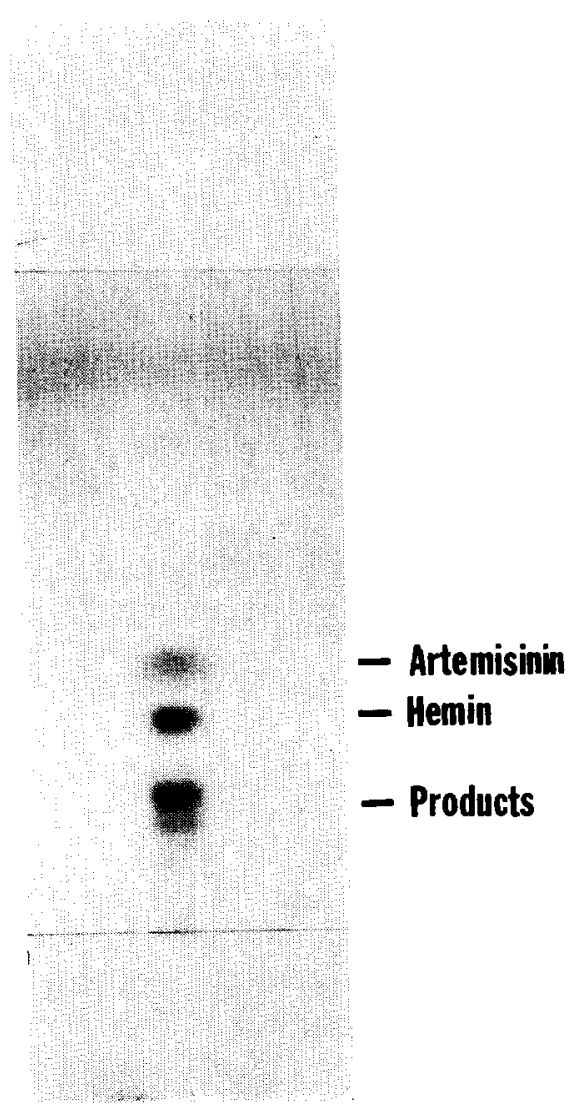

Fig. 3. TLC of artemisinin, hemin and the isolated hemin/artemisinin adducts visualized by exposure to iodine vapor.

is 0.41 and the $R f$ of hemin is 0.33 . The adducts have $R f$ s of 0.16 and 0.21 ]. Neither adduct is fluorescent, suggesting that they still contain iron atoms (data not shown). Unfortunately, however, we were unable to successfuly remove the iron from these adducts in order to be able to run NMRs.

TLC and HPLC were then used to determine whether these adducts were also formed in vivo. The TLC system used could easily distinguish between artemisinin, heme and the adducts, although not easily between the two adducts (Fig. 3) When hemozoin was isolated from $\left[{ }^{14} \mathrm{C}\right]$ artemisinin treated parasites and analyzed by TLC, $77 \%$ of the total radioactivity comigrated with the synthetic adducts (Fig. 4). Similar results were found using HPLC (Fig. 5), which also could

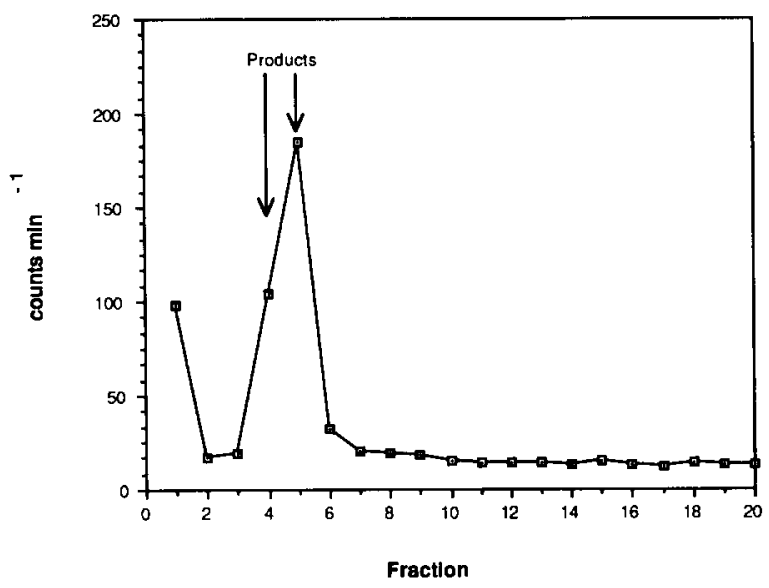

Fig. 4. Radioactivity profile of TLC run on hemozoin isolated from $\left[{ }^{14} \mathrm{C}\right]$ artemisinin-treated parasites. The parasites were cultured in the presence of $\left[{ }^{14} \mathrm{C}\right]$ artemisinin $\left(1.8 \mathrm{nCi} \mathrm{mi}^{-1}\right)$ for $6 \mathrm{~h}$ before isolation. Origin of plate on left, solvent front on right.

not distinguish easily between the two products (Fig. 1).

Artemisinin also reacted with isolated hemozoin. When $\left[{ }^{14} \mathrm{C}\right]$ artemisinin and hemozoin were incubated together, $\left[{ }^{14} \mathrm{C}\right]$ artemisinin disappears from solution in a time-dependent manner (Fig. 6). TLCs show a radioactive band, representing

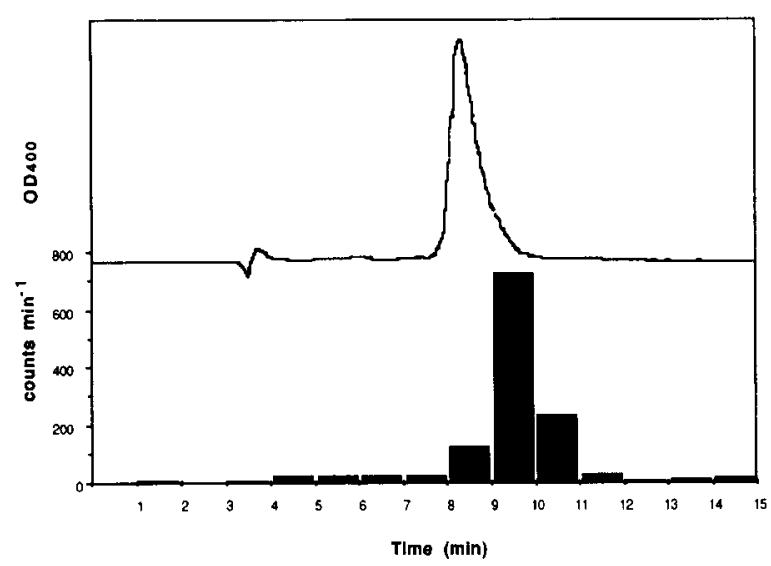

Fig. 5. HPLC profile of purified product $A$ (top) and of radioactivity eluted from an HPLC separation of hemozoin isolated from $\left[{ }^{14} \mathrm{C}\right]$ artemisinin treated parasites (bottom). Parasites were exposed to drug as in Table 1 and chromatographed on an analytical column as described in the Materials and Methods. Bottom graph shifted to the left by one minute to compensate for time delay between UV detector and fraction collector. 


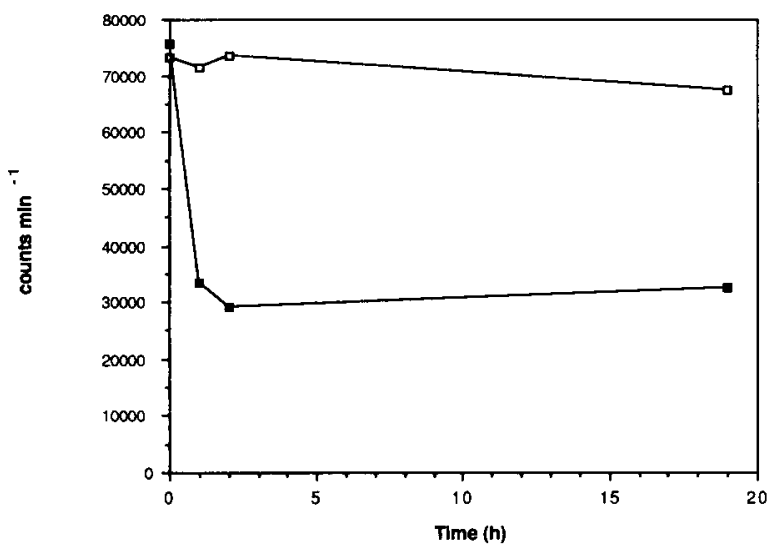

Fig. 6. Radioactivity in the supernatant during the incubation of $\left[{ }^{14} \mathrm{C}\right]$ artemisinin in the presence $(\square)$ and absence $(\square)$ of isolated hemozoin.

$20 \%$ of the hemozoin-associated radioactivity which comigrated with the adducts (not shown). This is the equivalent of incorporating $0.5 \mathrm{mmol}$ of artemisinin per mol of heme.

\section{Discussion}

Artemisinin is taken up by malaria parasites and concentrated in hemozoin, possibly by forming adducts. In aqueous solution, artemisinin was found to react with hemin, yielding two major products with molecular weights of 856 and 871 . Compounds with identical mobilities on TLC and HPLC were found in hemozoin, when either whole infected red cells of isolated hemozoin was incubated with drug. Thus, artemisinin-heme adducts appear to form in vivo.

The data presented here is consistent with a two-step mode of action of artemisinin, in which the first step consists of the heme-catalyzed cleavage of the endoperoxide bridge to form a free radical and the second step consists of the alkylation of heme and proteins by the drug-derived free radical [15] or other transient drug-derived intermediate [16]. Other evidence consistent with this mechanism includes observations that the endoperoxide bridge is necessary for biological activity $[17,18]$ and that free radical scavengers and iron chelators antagonize the drug [19-21]. The ability of heme and iron to catalyze the decomposition of artemisinin has been demonstrated by cyclic voltametry [7], by electron paramagnetic resonance spectroscopy [8] and by the isolation and characterization of the decomposition products [16]. Evidence for alkylation of heme [6] and proteins [9] has also been presented previously. This paper is the first time that the heme-artemisinin adducts have been isolated and partially characterized. The synthetic adducts are likely to be identical to the products formed when parasites are treated with ${ }^{14} \mathrm{C}$-labeled drug because they coelute on both silica TLC and reverse-phase HPLC. The importance of this process is underscored by the observation that about half of the cell-associated radiolabeled drug can be found in SDS-washed, protein-free hemozoin.

In a previous publication, we showed that a crude reaction mixture of heme and artemisinin, when analyzed by FAB mass spectrometry, gave peaks with $m / z$ values of $840,868,886$ and 914 [6]. In contrast, the purified peaks shown here give $m / z$ values of 856 and 871 by both ESI and FAB mass spectrometry. The differences between the two observations may be due to differences in the reaction conditions, as well as to the possibility that the impure reaction mixture contained components which were not observed by HPLC since the eluate was monitored at $400 \mathrm{~nm}$, a wavelength which is characteristic of compounds containing porphyrin rings [14]. Interestingly the $\mathrm{MW}$ of adduct $\mathrm{B}, 871$, is 18 less than that of the sum of artemisinin (282.4) and heme (616.5) suggesting a dehydration.

More artemisinin concentrates in hemozoin obtained from $\left[{ }^{14} \mathrm{C}\right]$ artemisinin-treated infected red cells ( $2.3 \mathrm{mmol} \mathrm{mol}{ }^{-1}$ heme) than by exposing isolated hemozoin to the labeled drug $(0.5 \mathrm{mmol}$ $\mathrm{mol}^{-1}$ heme). There are two possible reasons for this. First, the heme-catalyzed decomposition of artemisinin is reductive [16], suggesting that ferrous heme $\left(\mathrm{Fe}^{+2}\right)$ is more reactive than ferric heme $\left(\mathrm{Fe}^{+3}\right)$. Heme is more likely to be in the reduced state in vivo than in solution because of the presence of intracellular reducing agents such as glutathione. Second, in vivo, heme may react with artemisinin in the brief time period between when it is liberated from hemoglobin and poly- 
merized to hemozoin [4]. Once incorporated into hemozoin, only the heme molecules on the surface of the granules are likely to be reactive with artemisinin, since the iron atoms of the interior molecules may be sequestered by iron-carboxylate bonds [5].

What role does the formation heme-artemisinin adducts play in the drug-mediated killing of malaria parasites? The heme-artemisinin adduct is not toxic to in vitro cultures of $P$. falciparum when added to the media [6]. When parasites are grown in resealed red cells containing an excess of heme, they appear to be protected from artemisinin, presumably because the adducts form in the red cell cytoplasm (unpublished results). However, the CR strain of P. berghei, which lacks hemozoin, is resistant to artemisinin [22]. Furthermore, threequarters of the cell-associated drug winds up in hemozoin, of which two-thirds (half of the total) remains even after SDS extraction of protein. Thus, the formation of artemisinin-heme adducts play important but as yet undefined roles in the mode of action of this drug.

\section{Acknowledgements}

This work was supported by a grant from the UNDP/World Bank/WHO Special Programme for Research and Training in Tropical Diseases. We thank Dr. James Windak and Dr. Rachel Ogozalek Loo performing Mass Spectrometery, and Drs. Gary H. Posner and Paul Oritz De Montellano for helpful discussions.

\section{References}

[1] Klayman, D.L. (1985) Qinghaosu (artemisinin): an antimalarial drug from China, Science 228, 1049-1055.

[2] Qinghaosu Antimalaria Coordinating Research Group. (1979) Antimalaria studies on qinghaosu. Chin. Med. J. 92, $811-816$.

[3] Hien, T.T. and White, N.J. (1993) Qinghaosu. Lancet 341, 603-608.

[4] Goldberg, D.E., Slater, A.F.G., Cerami, A. and Henderson, G.B. (1990) Hemoglobin degradation in the malaria parasite Plasmodium falciparum: an ordered process in a unique organelle. Proc. Natl. Acad. Sci. USA 87, 29312935 .
[5] Slater, A.F.G., Swiggard, W.J., Orton, B.R., Flitter, W.D., Goldberg, D.E., Cerami, A. and Hendenson, G.B. (1991) An iron-carboxylate bond links the heme units of malaria pigment. Proc. Natl. Acad. Sci. USA 88, 325-329.

[6] Meshnick, S.R., Thomas, A., Ranz, A., Xu, C.-M. and Pan, H.-Z. (1991) Artemisinin (qinghaosu): the role of intracellular hemin in its mechanism of antimalarial action. Mol. Biochem. Parasitol 49, 181-190.

[7] Zhang, F., Gosser, D. and Meshnick, S.R. (1992). Hemincatalyzed decomposition of artemisinin (qinghaosu). Biochem. Pharmacol. 43, 1805-1809.

[8] Meshnick, S.R., Yang, Y.Z., Lima, V., Kuyers, F., Kamchonwongpaisan, S. and Yuthavong, Y. (1993) Irondependent free radical generation from the antimalarial agent artemisinin (qinghaosu). Antimicrob. Agent Chemother. 37, 1108-1114

[9] Yang, Y.Z., Asawamahasakda, W. and Meshnick, S.R. (1993) Alkylation of human albumin by the antimalarial artemisinin. Biochem. Pharmacol. 46, 336-339.

[10] Trager. W. and Jensen, J.B. (1976) Human malaria parasites in continuous culture. Science 193, 673-675.

[11] Goldie, P., Roth, E.F., Jr., Oppenheim, J. and Vanderberg, J.P. (1990) Biochemical characterization of Plasmodium falciparum hemozoin. Am. J. Trop. Med. Hyg. 43, 584 596.

[12] Choe, Y.S. and Ortiz de Montellano, P.R. (1991) Differential additions to the myoglobin prosthetic heme group. J. Bio. Chem. 266, 8523-8530.

[13] Smith, P.K., Krohn, R.I., Hermanson, G.T., Mallia, A.K., Gartner, F.H., Provenzano, M.D., Fujimoto, E.K., Goeke, N.M., Olson, B.J. and Klenk, D.C. (1985) Measurement of protein using bicinchoninic acid. Anal. Biochem. 150: 7685.

[14] Fuhrhop, J.H. and Smith, K.M. (1975) Estimation of porphyrins and metalloporphyrins, In: Porphyrins and Metalloporphyrins, Smith K.M. (ed.), pp. 804-807. Elsevier, Amsterdam.

[15] Meshnick, S.R. (In Press) The mode of action of antimalarial endoperoxides. Trans. R. Soc. Trop. Med. Hyg.

[16] Posner, G.H. and Oh, C.H. (1992) A regiospecifically oxygen-18 labeled 1,2,4-trioxane: a simple chemical model system to probe the mechanism(s) for the antimalarial activity of artemisinin (qinghaosu). J. Am. Chem. Soc. 114, 8328-8329.

[17] Brossi, A., Venugopalan, B., Gerpe, L.D., Yeh, H.J.C., Flippen-Anderson, J.L., Buchs, P., Luo, X.D., Milhous, W. and Peters, W. (1988) Arteether, a new antimalarial drug: synthesis and antimalarial properties. J. Med. Chem. 31, 645-650.

[18] China Cooperative Research Group on Qinghaosu and Its Derivatives as Antimalarials (1982) Chemical studies on qinghaosu (artemisinine). J. Traditional Chin. Med. 2, 3-8.

[19] Krungkrai, S.R. and Yuthavong, Y. (1987) The antimalarial action of qinghaosu and artesunate in combination with agents that modulate oxidant stress. Trans. R. Soc. Trop. Med. Hyg. 81, 710-714. 
[20] Levander, O.A., Ager, A.L., Morris, V.C. and May, R.G. (1989) Qinghaosu, dietary vitamin E, selenium andcodliver oil: effect on the susceptibility of mice to the malarial parasite Plasmodium yoelii. Am. J. Clin. Nutr. 50, 346-352.

[21] Meshnick, S.R., Tsang, T.W., Lin, F.B., Pan, H.Z., Chang, Z.N., Kuyper, F., Chiu, D. and Lubin, B. (1989) Activated oxygen mediates the antimalarial activity of qinghaosu. Prog. Clin. Biol. Res. 313, 95-104.

[22] Peters, W., L. Lin, B.L. Robinson and D.C. Warhurst. (1986) The chemotherapy of rodent malaria, XL. The action of artemisinin and related sesquiterpenes. Ann. Trop. Med. Hyg. 80, 483-489. 